KYUNGPOOK Math. J. 48(2008), 93-100

\title{
A New Hilbert-type Integral Inequality with Some Parame- ters and Its Reverse
}

\section{Zitian XIE}

Dept. of Math. Zhaoqing University, Zhaoqing, Guangdong, 526061, P. R. China e-mail : gdzqxzt@163.com

BICHENG YANG

Dept. of Math. Guangdong Education Institute, Guangzhou, Guangdong, 510303, P. R. China

e-mail : bcyang@pub.guangzhou.gd.cn

ABstract. In this paper, by introducing some parameters and estimating the weight function, we give a new Hilbert-type integral inequality with a best constant factor. The equivalent inequality and the reverse forms are considered.

\section{Introduction}

If $f(x), g(x) \geq 0$, such that $0<\int_{0}^{\infty} f^{2}(x) d x<\infty$ and $0<\int_{0}^{\infty} g^{2}(x) d x<\infty$, then (see [1])

$$
\int_{0}^{\infty} \int_{0}^{\infty} \frac{f(x) g(x)}{x+y} d x d y<\pi\left\{\int_{0}^{\infty} f^{2}(x) d x \int_{0}^{\infty} g^{2}(x) d x\right\}^{1 / 2},
$$

where the constant factor $\pi$ is the best possible. Inequality (1.1) is well-known as Hilbert's integral inequality, which had been extended by Hardy-Riesz as (see [2]):

If $p>1, \frac{1}{p}+\frac{1}{q}=1, f(x), g(x) \geq 0$, such that $0<\int_{0}^{\infty} f^{p}(x) d x<\infty$ and $0<\int_{0}^{\infty} g^{q}(x) d x<\infty$, then we have the following Hardy-Hilbert's integral inequality:

$$
\int_{0}^{\infty} \int_{0}^{\infty} \frac{f(x) g(y)}{x+y} d x d y<\frac{\pi}{\sin (\pi / p)}\left\{\int_{0}^{\infty} f^{p}(x) d x\right\}^{1 / p}\left\{\int_{0}^{\infty} g^{q}(x) d x\right\}^{1 / q}
$$

where the constant factor $\frac{\pi}{\sin (\pi / p)}$ is the best possible.

Hardy-Hilbert's integral inequality is important in analysis and its applications (see [3]). In recent years, Yang [4], [5] gave two different best generalizations of (1.2) by introducing a parameter $\lambda>0$, and Yang et al. [6] gave an extension of the above

Received August 28, 2006.

2000 Mathematics Subject Classification: 11Y05, 11A51, 11 B39.

Key words and phrases: Hilbert-type integral inequality, weight function; Hölder's inequality; equivalent inequality, reverse form. 
results by introducing the index of conjugate parameter $(r, s)\left(r>1, \frac{1}{r}+\frac{1}{s}=1\right)$ as follows (see [6],(21) for $\mathrm{n}=2$ ):

If $f(x), g(x) \geq 0$, and $0<\int_{0}^{\infty} x^{p\left(1-\frac{\lambda}{r}\right)-1} f^{p}(x) d x<\infty, 0<\int_{0}^{\infty} x^{q\left(1-\frac{\lambda}{s}\right)-1} g^{q}(x) d x<$ $\infty$, then

$$
\begin{aligned}
& \int_{0}^{\infty} \int_{0}^{\infty} \frac{f(x) g(y)}{(x+y)^{\lambda}} d x d y \\
< & B\left(\frac{\lambda}{r}, \frac{\lambda}{s}\right)\left\{\int_{0}^{\infty} x^{p\left(1-\frac{\lambda}{r}\right)-1} f^{p}(x) d x\right\}^{\frac{1}{p}}\left\{\int_{0}^{\infty} x^{q\left(1-\frac{\lambda}{s}\right)-1} g^{q}(x) d x\right\}^{\frac{1}{q}},
\end{aligned}
$$

where the constant factor $B\left(\frac{\lambda}{r}, \frac{\lambda}{s}\right)$ is the best possible $(\mathrm{B}(\mathrm{u}, \mathrm{v})$ is the Beta function). In particular, for $\lambda=1, r=q, s=p$, inequality (1.3) reduces to (1.2); for $\lambda=4, r=$ $s=2,(1.3)$ reduces to:

$$
\int_{0}^{\infty} \int_{0}^{\infty} \frac{f(x) g(y)}{(x+y)^{4}} d x d y<\frac{1}{6}\left\{\int_{0}^{\infty} \frac{1}{x^{p+1}} f^{p}(x) d x\right\}^{\frac{1}{p}}\left\{\int_{0}^{\infty} \frac{1}{x^{q+1}} g^{q}(x) d x\right\}^{\frac{1}{q}}
$$

In this paper, by introducing some parameters and estimating the weight function, we prove a new Hilbert-type integral inequality with a best constant factor similar to (1.4). The equivalent inequality and the reverse forms are considered.

\section{Some lemmas}

Lemma 2.1. If $a, b>0, a \neq b$, define the weight functions $\varpi(x)$ and $\omega(y)$ as

$$
\begin{aligned}
& \varpi(x)=\int_{0}^{\infty} \frac{x^{2} y}{(x+a y)^{2}(x+b y)^{2}} d y(x \in(0, \infty)) ; \\
& \omega(y)=\int_{0}^{\infty} \frac{x y^{2}}{(x+a y)^{2}(x+b y)^{2}} d x(y \in(0, \infty)),
\end{aligned}
$$

then we have

$$
\varpi(x)=\omega(y)=K:=\frac{a+b}{(b-a)^{2}}\left[\frac{\ln (b / a)}{b-a}-\frac{2}{a+b}\right] .
$$

Proof. For fixed $\mathrm{x}$, setting $u=y / x$ in (2.1), we obtain

$$
\begin{aligned}
\varpi(x) & =\int_{0}^{\infty} \frac{u}{(1+a u)^{2}(1+b u)^{2}} d u \\
& =\frac{1}{(b-a)^{2}}\left[\frac{1}{1+a u}+\frac{1}{1+b u}\right]_{0}^{\infty}-\left[\frac{a+b}{(b-a)^{3}} \ln \left(\frac{1+a u}{1+b u}\right)\right]_{0}^{\infty} \\
& =\frac{1}{(b-a)^{2}}\left[-2+(a+b) \frac{\ln (b / a)}{b-a}\right] .
\end{aligned}
$$


Hence we obtain $\varpi(x)=K$. In the same way, we obtain $\omega(y)=K$. The lemma is proved.

Lemma 2.2. If $a, b>0$ and $a \neq b$, then for $0<\varepsilon<p$, we have

$$
\int_{0}^{\infty} \frac{u^{1-\varepsilon / p}}{(1+a u)^{2}(1+b u)^{2}} d u=K+o(1)\left(\varepsilon \rightarrow 0^{+}\right)
$$

Proof. Since for $a, b>0$ and $a \neq b$, we find

$$
\begin{aligned}
& \left|\int_{0}^{\infty} \frac{u^{1-\varepsilon / p}}{(1+a u)^{2}(1+b u)^{2}} d u-K\right|=\left|\int_{0}^{\infty} \frac{u^{1-\varepsilon / p}-u}{(1+a u)^{2}(1+b u)^{2}} d u\right| \\
\leq & \int_{0}^{1} \frac{\left|u^{1-\varepsilon / p}-u\right|}{(1+a u)^{2}(1+b u)^{2}} d u+\int_{1}^{\infty} \frac{\left|u^{1-\varepsilon / p}-u\right|}{(1+a u)^{2}(1+b u)^{2}} d u . \\
\leq & \int_{0}^{1}\left(u^{1-\varepsilon / p}-u\right) d u+\frac{1}{(a b)^{2}} \int_{1}^{\infty} \frac{u-u^{1-\varepsilon / p}}{u^{4}} d u \\
= & \left(\frac{1}{2-\varepsilon / p}-\frac{1}{2}\right)+\frac{1}{(a b)^{2}}\left(\frac{1}{2}-\frac{1}{2+\varepsilon / p}\right) \rightarrow 0, \quad \text { for } \varepsilon \rightarrow 0^{+} .
\end{aligned}
$$

Then (2.4) is valid, and the lemma is proved.

Lemma 2.3. If $p>1$ (or $0<p<1$ ), $\frac{1}{p}+\frac{1}{q}=1, a, b>0, a \neq b$, and $0<\varepsilon<p$, setting

$$
I:=\int_{1}^{\infty}\left[\int_{1}^{\infty} \frac{y^{1-\varepsilon / p}}{(x+a y)^{2}(x+b y)^{2}} d y\right] x^{1-\varepsilon / q} d x,
$$

then we have

$$
\frac{1}{\varepsilon}(K+o(1))-O(1) \leq I \leq \frac{1}{\varepsilon}(K+o(1)), \varepsilon \rightarrow 0^{+} .
$$

Proof. For fixed $x$, setting $y=x u$, then by (2.4), we obtain

$$
\begin{aligned}
I= & \int_{1}^{\infty} x^{-1-\varepsilon}\left[\int_{x^{-1}}^{\infty} \frac{u^{1-\varepsilon / p}}{(1+a u)^{2}(1+b u)^{2}} d u\right] d x \\
= & \int_{1}^{\infty} x^{-1-\varepsilon}\left[\int_{0}^{\infty} \frac{u^{1-\varepsilon / p}}{(1+a u)^{2}(1+b u)^{2}} d u\right] d x \\
& \quad-\int_{1}^{\infty} x^{-1-\varepsilon}\left[\int_{0}^{x^{-1}} \frac{u^{1-\varepsilon / p}}{(1+a u)^{2}(1+b u)^{2}} d u\right] d x \\
\geq & \frac{1}{\varepsilon}(K+o(1))-\frac{1}{a+b} \int_{1}^{\infty} x^{-1}\left(\int_{0}^{x^{-1}} u^{-\frac{\varepsilon}{p}} d u\right) d x\left((1+a u)^{2}(1+b u)^{2}>(a+b) u\right) \\
= & \frac{1}{\varepsilon}(K+o(1))-\frac{1}{a+b}\left(1-\frac{\varepsilon}{p}\right)^{-2}=\frac{1}{\varepsilon}(K+o(1))-O(1) .
\end{aligned}
$$


By the same way, we have

$$
I \leq \int_{1}^{\infty}\left[\int_{0}^{\infty} \frac{y^{1-\varepsilon / p}}{(x+a y)^{2}(x+b y)^{2}} d y\right] x^{1-\varepsilon / q} d x=\frac{1}{\varepsilon}(K+o(1)) .
$$

The lemma is proved.

\section{Main results}

Theorem 3.1. If $p>1, \frac{1}{p}+\frac{1}{q}=1, a, b>0, a \neq b, f(x), g(x) \geq 0$, such that $0<\int_{0}^{\infty} \frac{1}{x^{p+1}} f^{p}(x) d x<\infty$ and $0<\int_{0}^{\infty} \frac{1}{x^{q+1}} g^{q}(x) d x<\infty$, then

$$
\begin{aligned}
& \int_{0}^{\infty} \int_{0}^{\infty} \frac{f(x) g(y)}{(x+a y)^{2}(x+b y)^{2}} d x d y \\
< & K\left\{\int_{0}^{\infty} \frac{1}{x^{p+1}} f^{p}(x) d x\right\}^{\frac{1}{p}}\left\{\int_{0}^{\infty} \frac{1}{x^{q+1}} g^{q}(x) d x\right\}^{\frac{1}{q}},
\end{aligned}
$$

where the constant factor $K$ is the best possible and $K$ is defined by (2.3).

Theorem 3.2. If $0<p<1, \frac{1}{p}+\frac{1}{q}=1, a, b>0, a \neq b, f(x), g(x) \geq 0$, such that $0<\int_{0}^{\infty} \frac{1}{x^{p+1}} f^{p}(x) d x<\infty$ and $0<\int_{0}^{\infty} \frac{1}{x^{q+1}} g^{q}(x) d x<\infty$, then

$$
\begin{aligned}
& \int_{0}^{\infty} \int_{0}^{\infty} \frac{f(x) g(y)}{(x+a y)^{2}(x+b y)^{2}} d x d y \\
> & K\left\{\int_{0}^{\infty} \frac{1}{x^{p+1}} f^{p}(x) d x\right\}^{\frac{1}{p}}\left\{\int_{0}^{\infty} \frac{1}{x^{q+1}} g^{q}(x) d x\right\}^{\frac{1}{q}},
\end{aligned}
$$

where the constant factor $K$ is the best possible.

Proof of Theorem 3.1. By Hölder's inequality with weight (see [7]) and (2.1)-(2.3), we have,

$$
\begin{aligned}
J:= & \int_{0}^{\infty} \int_{0}^{\infty} \frac{f(x) g(y)}{(x+a y)^{2}(x+b y)^{2}} d x d y \\
= & \int_{0}^{\infty} \int_{0}^{\infty} \frac{1}{(x+a y)^{2}(x+b y)^{2}}\left[\left(\frac{y^{1 / p}}{x^{1 / q}}\right) f(x)\right]\left[\left(\frac{x^{1 / q}}{y^{1 / p}}\right) g(y)\right] d x d y \\
\leq & \left\{\int_{0}^{\infty} \int_{0}^{\infty} \frac{1}{(x+a y)^{2}(x+b y)^{2}}\left(\frac{y}{x^{p-1}}\right) f^{p}(x) d y d x\right\}^{\frac{1}{p}} \\
& \times\left\{\int_{0}^{\infty} \int_{0}^{\infty} \frac{1}{(x+a y)^{2}(x+b y)^{2}}\left(\frac{x}{y^{q-1}}\right) g^{q}(y) d x d y\right\}^{\frac{1}{q}} \\
= & \left\{\int_{0}^{\infty} \varpi(x) \frac{1}{x^{p+1}} f^{p}(x) d x\right\}^{\frac{1}{p}}\left\{\int_{0}^{\infty} \omega(y) \frac{1}{y^{q+1}} g^{q}(y) d x\right\}^{\frac{1}{q}} \\
= & K\left\{\int_{0}^{\infty} \frac{1}{x^{p+1}} f^{p}(x) d x\right\}^{1 / p}\left\{\int_{0}^{\infty} \frac{1}{x^{q+1}} g^{q}(x) d x\right\}^{1 / q} .
\end{aligned}
$$


If (3.3) takes the form of equality, then there exists constants $M$ and $N$, such that they are not all zero, and (see [7])

$$
M\left(\frac{y}{x^{p-1}}\right) f^{p}(x)=N\left(\frac{x}{y^{q-1}}\right) g^{q}(y) \quad \text { a.e. in }(0, \infty) \times(0, \infty) .
$$

Hence, there exists a constant $C$, such that

$$
M x^{-p} f^{p}(x)=N y^{-q} g^{q}(y)=C \quad \text { a.e. in } \quad(0, \infty) .
$$

We claim that $M=0$. In fact, if $M \neq 0$, then $x^{-1-p} f^{p}(x)=C /(M x)$ a.e. in $(0, \infty)$, which contradicts the fact that $0<\int_{0}^{\infty} x^{-1-p} f^{p}(x) d x<\infty$. In the same way, we claim that $N=0$. This is a contradiction. Hence by (3.3), we have (3.1).

If the constant factor $K$ in (3.1) is not the best possible, then there exists a positive constant $H$ (with $H<K$ ), such that (3.1) is still valid if we replace $K$ by $H$. For $0<\varepsilon<p$ small enough, setting $f_{\varepsilon}$ and $g_{\varepsilon}$ as $f_{\varepsilon}(x)=g_{\varepsilon}(x)=0$, for $x \in(0,1) ; f_{\varepsilon}(x)=x^{1-\varepsilon / p} ; g_{\varepsilon}(x)=x^{1-\varepsilon / q}$, for $x \in[1, \infty)$, then we obtain

$$
\begin{aligned}
& H\left\{\int_{0}^{\infty} \frac{1}{x^{p+1}} f_{\varepsilon}^{p}(x) d x\right\}^{1 / p}\left\{\int_{0}^{\infty} \frac{1}{x^{q+1}} g_{\varepsilon}^{q}(x) d x\right\}^{1 / q} \\
= & H\left\{\int_{1}^{\infty} x^{-\varepsilon-1} d x\right\}^{1 / p}\left\{\int_{1}^{\infty} x^{-\varepsilon-1} d x\right\}^{1 / q}=\frac{H}{\varepsilon} .
\end{aligned}
$$

By (2.5), we have

$$
\begin{aligned}
& \int_{0}^{\infty} \int_{0}^{\infty} \frac{f_{\varepsilon}(x) g_{\varepsilon}(y)}{(x+a y)^{2}(x+b y)^{2}} d x d y \\
= & \int_{1}^{\infty}\left[\int_{1}^{\infty} \frac{y^{1-\frac{\varepsilon}{q}}}{(x+a y)^{2}(x+b y)^{2}} d y\right] x^{1-\frac{\varepsilon}{p}} d x=I \\
\geq & \frac{1}{\varepsilon}(K+o(1))-O(1) .
\end{aligned}
$$

Hence we find

$$
\frac{1}{\varepsilon}(K+o(1))-O(1)<\frac{H}{\varepsilon} \text { or }(K+o(1))-\varepsilon O(1)<H .
$$

For $\varepsilon \rightarrow 0^{+}$, it follows that $K \leq H$. This contradicts the fact that $H<K$. Hence the constant factor $K$ in (3.1) is the best possible. The theorem is proved.

Proof of Theorem 3.2. By the reverse Hölder's inequality with weight (see [7]) and the same way of giving (3.3), we obtain (3.2).

If the constant factor $K$ in (3.2) is not the best possible, then there exists a positive constant $H$ (with $H>K$ ), such that (3.2) is still valid if we replace $K$ by $H$. For $0<\varepsilon<p$ small enough, setting $f_{\varepsilon}$ and $g_{\varepsilon}$ as: $f_{\varepsilon}(x)=g_{\varepsilon}(x)=0$, for 
$x \in(0,1) ; f_{\varepsilon}(x)=x^{1-\varepsilon / p} ; g_{\varepsilon}(x)=x^{1-\varepsilon / q}$, for $x \in[1, \infty)$, then we obtain

$$
\begin{aligned}
& H\left\{\int_{0}^{\infty} \frac{1}{x^{p+1}} f_{\varepsilon}^{p}(x) d x\right\}^{1 / p}\left\{\int_{0}^{\infty} \frac{1}{x^{q+1}} g_{\varepsilon}^{q}(x) d x\right\}^{1 / q} \\
= & H\left\{\int_{1}^{\infty} x^{-\varepsilon-1} d x\right\}^{1 / p}\left\{\int_{1}^{\infty} x^{-\varepsilon-1} d x\right\}^{1 / q}=\frac{H}{\varepsilon} .
\end{aligned}
$$

By (2.5), we have

$$
\begin{aligned}
& \int_{0}^{\infty} \int_{0}^{\infty} \frac{f_{\varepsilon}(x) g_{\varepsilon}(y)}{(x+a y)^{2}(x+b y)^{2}} d x d y \\
= & \int_{1}^{\infty}\left[\int_{1}^{\infty} \frac{y^{1-\frac{\varepsilon}{p}}}{(x+a y)^{2}(x+b y)^{2}} d y\right] x^{1-\frac{\varepsilon}{q}} d x=I \\
\leq & \frac{1}{\varepsilon}(K+o(1)) .
\end{aligned}
$$

Hence we find

$$
\frac{1}{\varepsilon}(K+o(1))>\frac{H}{\varepsilon} \text { or }(K+o(1))>H .
$$

For $\varepsilon \rightarrow 0^{+}$, it follows that $K \geq H$. This contradicts the fact that $H>K$. Hence the constant $K$ in (3.2) is the best possible. The theorem is proved.

Theorem 3.3. Under the same assumption of Theorem 3.1. we have

$$
\int_{0}^{\infty} y^{2 p-1}\left[\int_{0}^{\infty} \frac{f(x)}{(x+a y)^{2}(x+b y)^{2}} d x\right]^{p} d y<K^{p} \int_{0}^{\infty} \frac{1}{x^{p+1}} f^{p}(x) d x,
$$

where the constant factor $K^{p}$ is the best possible. Inequalities (3.5) and (3.1) are equivalent.

Theorem 3.4. Under the same assumption of Theorem 3.2. we have

$$
\int_{0}^{\infty} y^{2 p-1}\left[\int_{0}^{\infty} \frac{f(x)}{(x+a y)^{2}(x+b y)^{2}} d x\right]^{p} d y>K^{p} \int_{0}^{\infty} \frac{1}{x^{p+1}} f^{p}(x) d x,
$$

where the constant factor $K^{p}$ is the best possible. Inequalities (3.6) and (3.2) are equivalent.

We prove only Theorem 3.3, since the proof of Theorem 3.4 is the similar.

Proof. Setting $g(y)=y^{2 p-1}\left[\int_{0}^{\infty} \frac{f(x)}{(x+a y)^{2}(x+b y)^{2}} d x\right]^{p-1}$, by (3.1), we have

$$
\begin{aligned}
& \int_{0}^{\infty} y^{-1-q} g^{q}(y) d y=\int_{0}^{\infty} y^{2 p-1}\left[\int_{0}^{\infty} \frac{f(x)}{(x+a y)^{2}(x+b y)^{2}} d x\right]^{p} d y \\
= & J \leq K\left\{\int_{0}^{\infty} \frac{1}{x^{p+1}} f^{p}(x) d x\right\}^{\frac{1}{p}}\left\{\int_{0}^{\infty} \frac{1}{x^{q+1}} g^{q}(x) d x\right\}^{\frac{1}{q}}
\end{aligned}
$$




$$
0<\left\{\int_{0}^{\infty} \frac{1}{y^{q+1}} g^{q}(y) d y\right\}^{1 / p} \leq K\left\{\int_{0}^{\infty} \frac{1}{x^{p+1}} f^{p}(x) d x\right\}^{1 / p}<\infty
$$

Hence by (3.1), both (3.7) and (3.8) preserve the form of strict inequalities, and we have (3.5).

By Hölder's inequality, we have

$$
\begin{aligned}
J= & \int_{0}^{\infty}\left[y^{1+1 / q} \int_{0}^{\infty} \frac{f(x)}{(x+a y)^{2}(x+b y)^{2}} d x\right]\left(y^{-1-1 / q} g(y)\right) d y \\
\leq & \left\{\int_{0}^{\infty} y^{2 p-1}\left[\int_{0}^{\infty} \frac{f(x)}{(x+a y)^{2}(x+b y)^{2}} d x\right]^{p} d y\right\}^{\frac{1}{p}} \\
& \times\left(\int_{0}^{\infty} \frac{1}{y^{q+1}} g^{q}(y) d y\right)^{\frac{1}{q}} .
\end{aligned}
$$

Then by (3.5), we have (3.1). Hence inequalities (3.1) and (3.5) are equivalent.

If the constant factor in (3.5) is not the best possible, then by (3.9), we can get a contradiction that the constant factor in (3.1) is not the best possible. The theorem is proved.

Remark. Since we obtain

$$
\lim _{x \rightarrow a}\left\{\frac{x+a}{(x-a)^{2}}\left[\frac{\ln \left(\frac{x}{a}\right)}{x-a}-\frac{2}{x+a}\right]\right\}=\lim _{x \rightarrow a}\left[\frac{(x+a) \ln \left(\frac{x}{a}\right)-2(x-a)}{(x-a)^{3}}\right]=\frac{1}{6 a^{2}}
$$

then by (3.1), setting $b \rightarrow a$. we have the following new inequality:

$$
\begin{aligned}
& \int_{0}^{\infty} \int_{0}^{\infty} \frac{f(x) g(y)}{(x+a y)^{4}} d x d y \\
< & \frac{1}{6 a^{2}}\left\{\int_{0}^{\infty} \frac{1}{x^{p+1}} f^{p}(x) d x\right\}^{\frac{1}{p}}\left\{\int_{0}^{\infty} \frac{1}{x^{q+1}} g^{q}(x) d x\right\}^{\frac{1}{q}} .
\end{aligned}
$$

In particular, for $a=1,(3.11)$ reduces to (1.4). Hence we can get a conclusion that inequality (3.1) is a best extension of (1.4).

\section{References}

[1] G. H. Hardy, J. E.Littlewood and G. Pólya, Inequalities, Cambridge University Press, Cambridge, 1952.

[2] G. H. Hardy, Note on a theorem of Hilbert concerning series of positive terms, Proceedings London Math. Soc., Records of Proc. XLV-XLIV, 23(2)(1925).

[3] D. S. Mintrinović, J. E. Pečarić and A. M. Fink, Inequalities involving functions and their integrals and Derivertives, Kluwer Academic Publishers, Boston, 1991. 
[4] Bicheng Yang, On Hardy-Hilbert's integral inequality, J. Math. Anal. Appl., 261(2001), 295-306.

[5] Bicheng Yang, On the extended Hilbert's integral inequality, Journal of Inequalities in Pure and Applied Mathmatics, 5(4)(2004), Article 96.

[6] Bicheng Yang, Ilko Bnaetić, Mario Krnic and Josip Pečarić, Generalization of Hilbert and Hardy-Hilbert integral inequalities, Mathematical Inequalities and Applications, Mathematical Inequalities and Applications, 8(2)(2005), 259-272.

[7] Jichang Kang, Applied Inequalities, Shangdong Science and Technology Press, Jinan, 2004. 